\title{
The effect of fullerene soot on the mechanical properties of chitosan
}

\author{
O. Velazquez-Meraz ${ }^{1}$, A. Tejeda-Ochoa ${ }^{1}$, J. E. Ledezma-Sillas ${ }^{1}$, C. Carreño-Gallardo ${ }^{1}$, F. C. Robles- \\ Hernandez $^{2}$, J. M. Herrera-Ramirez ${ }^{1}$
}

${ }^{1}$ Centro de Investigación en Materiales Avanzados (CIMAV), Laboratorio Nacional de Nanotecnología, Miguel de Cervantes 120, 31136 Chihuahua, Chih., México.

${ }^{2}$ Department of Mechanical Engineering Technology, University of Houston, Houston, TX 77204-4020, USA.

Chitosan is a polysaccharide obtained by deacetylating chitin, which is the major constituent of the exoskeleton of crustaceous water animals [1]. When chitosan is reinforced by the addition of carbon species, its properties improve and therefore its application go beyond the usual, being an interesting topic in the fields of materials science and bioengineering [2]. By reinforcing chitosan through a suitable process that allows the reinforcer to be distributed and dispersed within the matrix, it will be an effective use to improve its mechanical properties.

This work proposes a synthesis based on a thermomechanical method, which involves the mechanical milling and conventional sintering processes to incorporate fullerene soot (FS) into the chitosan matrix. Mechanical milling produces highly homogenous nanostructured materials, improves integrity and has positive effects on mechanical properties [3]. Conventional sintering is proposed to preserve the nature of chitosan while improving upon the material properties.

The morphology of chitosan-FS composites was characterized by scanning electron microscopy (SEM) and bright field transmission electron microcopy (TEM). Mechanical properties (nanohardness and elastic modulus) were obtained by nanoindentation tests. During sintering, temperature was monitored via high speed, high resolution data acquisition system (NIcDAQ-9174: National Instruments, Austin, $\mathrm{TX})$. The collected information demonstrated the degradation of chitosan taking place above $220{ }^{\circ} \mathrm{C}$; with this information, sintering temperature criteria was applied with the following expression: $\mathrm{Ts}=(0.7-$ 0.8) $\mathrm{Tt}$, where $\mathrm{Tt}$ is the transformation temperature (in this case degradation temperature).

From Fig. 1 it can be seen that sintered chitosan samples experimented low cohesion among the chitosan particles, but this improved with the sintering conditions and the FS addition (Fig. 2). The addition of FS and temperature improves the sintering effectiveness by reducing grain boundaries and porosity. The TEM micrograph in Fig. 3 shows the interface between the FS and chitosan, where FS has graphitic nature having an interatomic distance of $0.35 \mathrm{~nm}$.

The nanohardness and elastic modulus results were obtained from the load-displacement curves (Fig. 4). The respective hardness values are 220 and $260 \mathrm{MPa}$ for samples sintered at $150{ }^{\circ} \mathrm{C}, 240$ and $300 \mathrm{MPa}$ for samples sintered at $180{ }^{\circ} \mathrm{C}$, which represent a 15 and $20 \%$ improvement, respectively. The elastic modulus results are 12.5 and $7 \mathrm{GPa}$ for samples sintered at $150{ }^{\circ} \mathrm{C}, 10.6$ and $5.4 \mathrm{GPa}$ for samples sintered at $180{ }^{\circ} \mathrm{C}$ that represent a 44 and $50 \%$ improvement in elastic behavior, respectively. It was demonstrated that the addition of $5 \mathrm{wt} \%$ of FS and sintering at $180{ }^{\circ} \mathrm{C}$ for $3 \mathrm{~h}$ enhanced the mechanical properties including elastic modulus and nanohardness, which is attributed to the improved cohesion among chitosan and FS as well as the grain structure refinement due to the mechanical milling. 


\section{References:}

[1] M. F. Goosen. Applications of chitin and chitosan. CRC Press (1996).

[2] C. Kumar. Biofunctionalization of nanomaterials (2005) p. 377.

[3] C. Suryanarayana. Progress in Materials Science 46(1) (2001) p. 1.
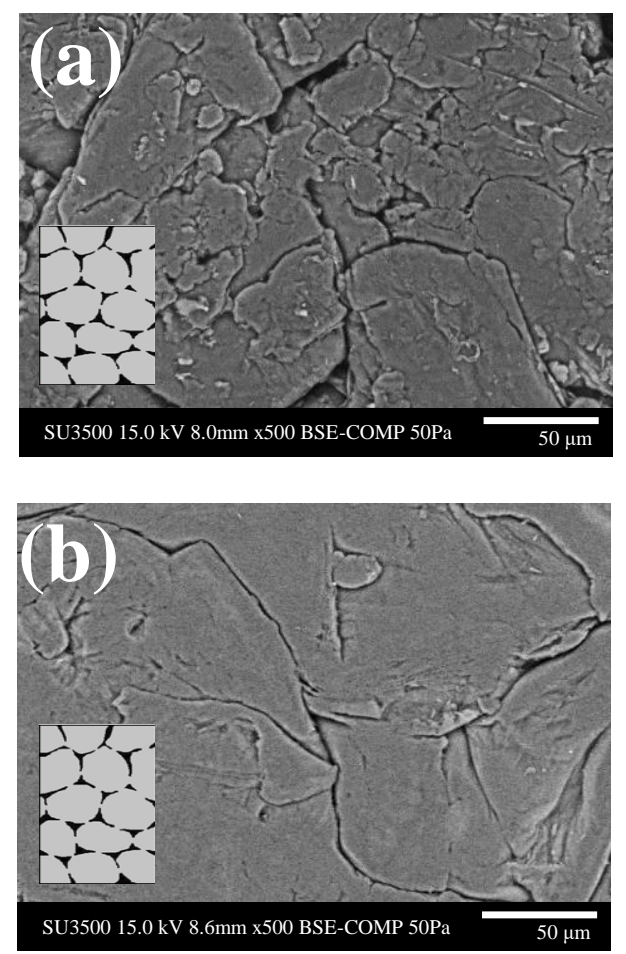

Fig. 1. SEM micrographs of chitosan sintered without reinforcement for $3 \mathrm{~h}$ at a) 150 and b) $180^{\circ} \mathrm{C}$.

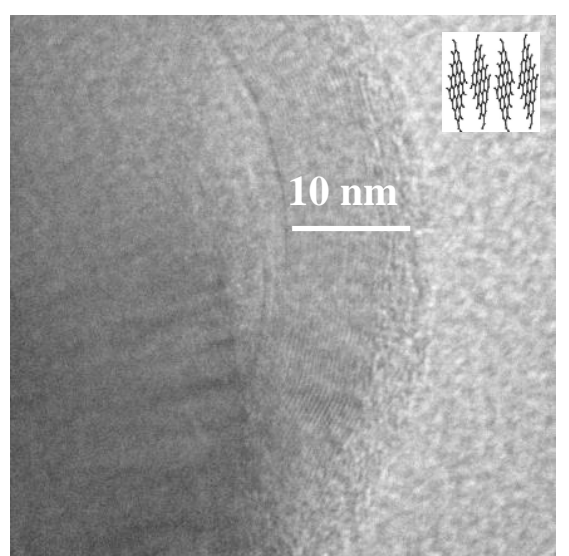

Fig. 3. TEM micrographs of chitosan-FS composites sintered for $3 \mathrm{~h}$ with $5 \mathrm{wt} \% \mathrm{FS}$ at $180^{\circ} \mathrm{C}$.
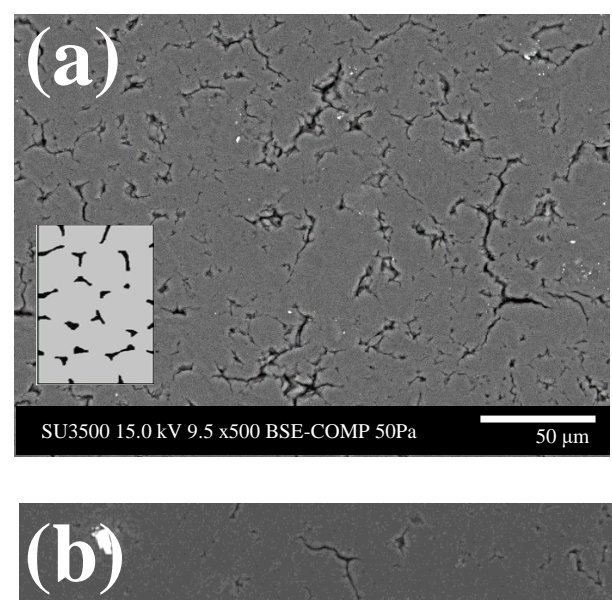

Fig. 2. SEM micrographs of chitosan-FS composites sintered for $3 \mathrm{~h}$ with $5 \mathrm{wt} \% \mathrm{FS}$ at a) 150 and b) $180^{\circ} \mathrm{C}$.

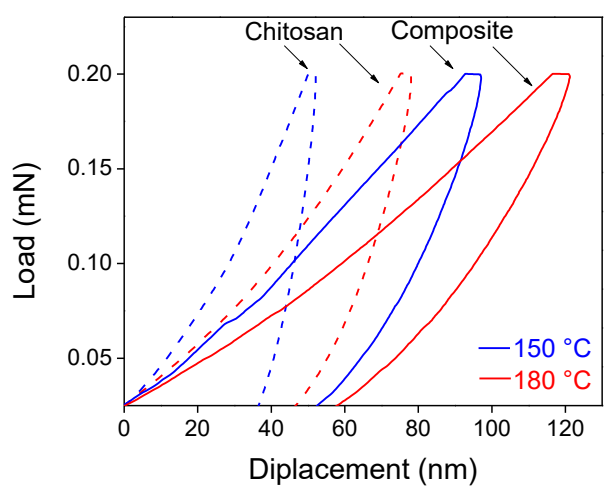

Fig. 4. Load-displacement curves for chitosan and chitosan-FS composites. 